\title{
Fiber-optic triggered release of liposome in vivo: implication of personalized chemotherapy
}

\author{
This article was published in the following Dove Press journal: \\ International Journal of Nanomedicine \\ 14 August 2015 \\ Number of times this article has been viewed
}

\author{
Huei-Ling Huang' \\ Pei-Hsuan Lu' \\ Hung-Chih Yang' \\ Gi-Da Lee ${ }^{1,2}$ \\ Han-Ru Li' \\ Kuo-Chih Liao' \\ 'Graduate Institute of Biomedical \\ Engineering, National Chung Hsing \\ University, ${ }^{2}$ Department of Radiology, \\ Taichung Veterans General Hospital, \\ Taichung, Taiwan
}

\begin{abstract}
The aim of this research is to provide proof of principle by applying the fiber-optic triggered release of photo-thermally responsive liposomes embedded with gold nanoparticles (AuNPs) using a $200 \mu \mathrm{m}$ fiber with $65 \mathrm{~mW}$ and $532 \mathrm{~nm}$ excitation for topical release in vivo. The tunable delivery function can be paired with an apoptosis biosensor based on the same fiber-optic configuration for providing real-time evaluation of chemotherapy efficacy in vivo to perform as a personalized chemotherapy system. The pattern of topical release triggered by laser excitation conveyed through optical fibers was monitored by the increase in fluorescence resulting from the dilution of self-quenching $(75 \mathrm{mM})$ fluorescein encapsulated in liposomes. In in vitro studies (in $37^{\circ} \mathrm{C}$ phosphate buffer saline), the AuNP-embedded liposomes showed a more efficient triggered release $(74.53 \% \pm 1.63 \%$ in 40 minutes) than traditional temperatureresponsive liposomes without AuNPs (14.53\% $\pm 3.17 \%$ ) or AuNP-liposomes without excitation $(21.92 \% \pm 2.08 \%)$ by spectroscopic measurements. Using the mouse xenograft studies, we first demonstrated that the encapsulation of fluorescein in liposomes resulted in a more substantial content retention $(81 \%)$ in the tumor than for free fluorophores $(14 \%)$ at 120 minutes after administration from in vivo fluorescence imaging. Furthermore, the preliminary results also suggested the tunable release capability of the system by demonstrating consecutive triggered releases with fiber-optic guided laser excitation.
\end{abstract}

Keywords: fiber-optic guided excitation, light excitation triggered release, photo-thermal responsive liposome, gold nanoparticles, tunable release in vivo

\section{Introduction}

Topical chemotherapy has proven valuable in locally advanced cancer treatment and potentially beneficial in metastatic patient management. As an adjuvant treatment of locally advanced cancer, it might reduce the tumor mass and enable more conservative surgery. ${ }^{1-3}$ It may also replace surgical tumor excision as the primary treatment in tumors with poor accessibility, ill-defined margins or that are attached to vital tissues. ${ }^{4}$ For metastatic patients, the removal of the primary tumor may improve therapeutic outcomes by prolonging survival, reducing the time to first progression, and reducing the occurrence of symptomatic chest wall diseases. ${ }^{5-7}$ One of the principal factors limiting therapeutic capacity is identifying the proper personalized dosing, a tradeoff between therapeutic efficiency and side effects (drug-related toxicities). Cytotoxic chemotherapy, which has a narrow therapeutic index between underdose and overdose, will still be the cornerstone in the following decades despite recent advances in anticancer development in the areas of immunotherapy, hormone therapy, and neovascular suppression with limited qualified candidates. ${ }^{3-8}$ However, the present means of clinical dosage calculation (by body surface area) is flawed and cannot reflect the actual elimination of the drug in situ for individual subjects, and exposes patients to the risk of underdosing (up to $30 \%$ for breast cancer) or overdosing. ${ }^{9}$
Correspondence: Kuo-Chih Liao Graduate Institute of Biomedical Engineering, National Chung Hsing University, 250 Kuo-Kuang Road, South District, Taichung 40227, Taiwan Tel +886 972 I 22873

Fax +886 422582422

Email kcliao@dragon.nchu.edu.tw
Dovepress

http://dx.doi.org/10.21 47/IJN.S85915
International Journal of Nanomedicine 20|5:|0 5|7|-5|85

(c) (1) \& $\odot 2015$ Huang et al. This work is published by Dove Medical Press Limited, and licensed under Creative Commons Attribution - Non Commercial (unported, v3.0)

5171 permission from Dove Medical Press Limited, provided the work is properly attributed. Permissions beyond the scope of the License are administered by Dove Medical Press Limited. Information on
por how to request permission may be found at: http://www.dovepress.com/permissions.php 
Liposomes are by far the most successful drug delivery vehicle in commercialization for clinical applications. ${ }^{10}$ For chemotherapy, the targeting (active or passive) and longlasting content release characteristics of liposome vesicles can facilitate the accumulation of a higher concentration/ percentage of the drug in the tumor (ie, increase the therapeutic index) and prevent the drug (toxic agent) from being exposed to normal tissues, which could cause lateral damage during transportation or allow it to be destroyed before arriving at the target tumor site. Liposome-delivered anticancer agents, such as doxorubicin, have been proved to reduce side effects (cardiotoxicity, gastrointestinal toxicity, stomatitis, hair loss, etc) while exhibiting superior performance or preserving efficacy in clinical studies and maintaining an extended period of therapeutic concentration. ${ }^{11-13}$ Considerable efforts have been devoted to extend the lifetime and integrity of liposomes in the bloodstream to periods on the order of days, raising the successful rate of transportation to the target location, ${ }^{14,15}$ but the liposomes interfere with the passive drug release efficiency upon arriving at the destination and reduce clinical efficacy. ${ }^{16-19}$ An active triggered mechanism is required to facilitate sufficient liposome content release in situ for optimal treatment effects, ${ }^{16-21}$ and a non-invasive or minimally invasive monitoring method is required to adjust the topical release kinetics tailored for each subject in vivo by detecting drug-induced bio-events, with the goal of achieving and extending the therapeutic dosage period without exceeding toxic levels. ${ }^{22-24}$

The conventional liposome triggered-release systems in development can be divided into external (remote) and internal (intrinsic) triggers. ${ }^{16,21}$ Internal triggers are designed to compromise liposome membrane integrity using biotransformable components resulting from elevated enzyme expression (phosphatase in prostatic carcinoma tissue or phospholipase $\mathrm{A}_{2}$ in breast or pancreatic cancer) or biophysical changes (endocytosed liposomes degraded by the endosomal pathway upon dropping $\mathrm{pH}$ from 6.5 to 4 ) at disease sites. External triggers convey external energy (ultrasound, heatgenerating light, microwaves or alternative magnetic fields) through tissues to focus on the target disease site and crush the accumulated liposomes in situ. However, both systems suffer from the inconsistency of subjects caused by in vivo fluctuations. Internal trigger systems could experience limited efficiency or delayed release due to highly variable in vivo biophysical changes $(\mathrm{pH}$, temperature, or chemical reactions competing with enzyme activity) and problematic specificity (phospholipase expressed not only in cancerous tissue but also in inflammation tissue). ${ }^{21}$ The energy input of external trigger systems could be significantly attenuated, reflected, and scattered during propagation through highly inhomogeneous and subject-dependent sophisticated bio-media, preventing it from attaining sufficient and consistent penetration in different subjects for clinical uses. ${ }^{16}$ The disadvantages of both conventional trigger methods are evidenced by the small number of animal studies demonstrating trigger release kinetics in vivo and the limited success in achieving adequate responses in vivo (for example, heat-generating methods cannot produce a uniform temperature increase in tumors with a diameter $>1 \mathrm{~cm}) .{ }^{17,18,25-27} \mathrm{In}$ addition, the release profiles of most triggers in development (internal and external) may be controversial because they are expected to rupture liposomes and cause a burst discharge. ${ }^{16,21}$ However, an ideal drug delivery system should steadily maintain the released therapeutic dosage (subject-dependent) for an extended period and prevent excess therapeutic agents (those which could not be absorbed by target cells or tissues) from diffusing out of the target site and resulting in lateral damage on exposed non-target tissue.

Our research group is developing a percutaneous sensing and therapeutic platform with a probe composed of hair-sized fiber bundles..$^{22-24,28,29}$ The probe can comprise optical fibers for conveying excitation light and detecting returning reflection or fluorescent emission, as well as microcapillaries for delivering biomarker diagnosing agents or therapeutic agents in batches or continuously by pneumatic pressure from a spritzer or mechanical pressure from a syringe pump. The first application is a personalized chemotherapy system with a tunable chemotherapy agent delivery function (Figure 1A) and an in vivo apoptosis monitoring sensing capability (Supplementary materials 1 and 2), both sharing the same fiber bundles for agent delivery and light transmission..$^{22}$ The experimental chemotherapy agent delivery platform delivers photo-thermally responsive liposomes directly into tumor, instead of relying on systematic intravenous administration. Dickerson et al demonstrated in a mouse study that intratumoral injection was more efficient at accumulating drugs in tumor tissue than intravenous administration. ${ }^{4}$ The proposed delivery system for personalized chemotherapy may also eliminate the inconsistency issue of non-invasive irradiation from conventional light sources, ${ }^{30}$ which was demonstrated in the study. The hair-like dimension of the implantable probe enables conventional clinical imaging modalities such as guided needle insertion (a similar procedure to biopsy sampling) to be used to place the fiber bundle probe within reach of deeper tumors (Figure 1C).

The aim of this research is to provide proof of principle by applying the fiber-optic triggered release of photo-thermally 
A

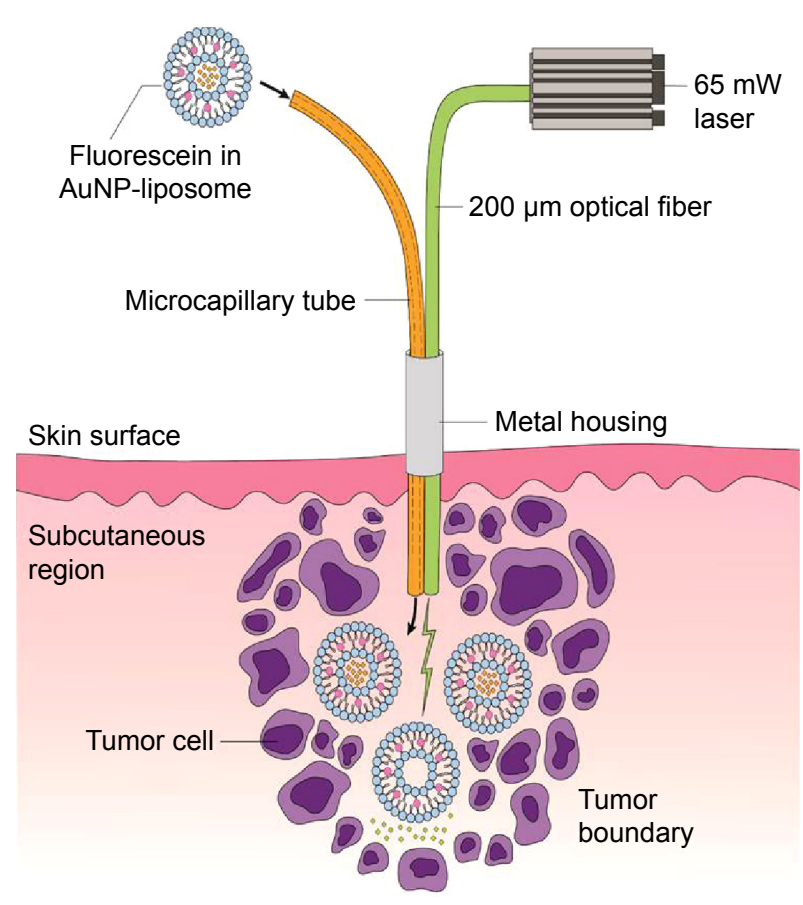

B

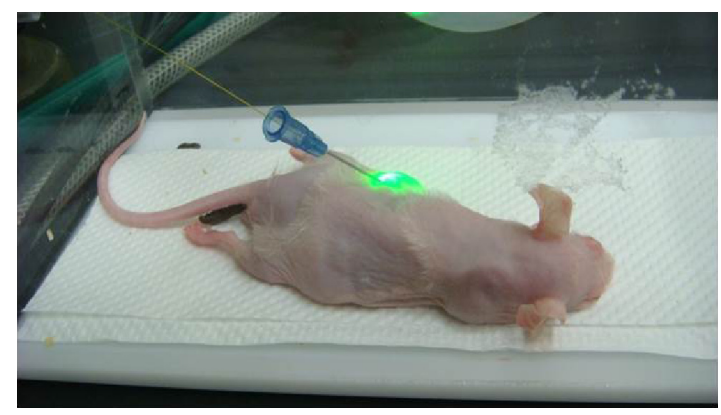

C

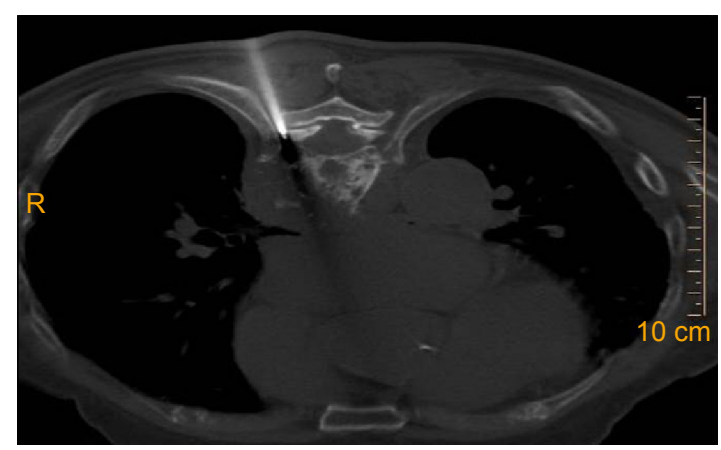

Figure I Concept of fiber-optic triggered photo-thermal responsive liposomes release in vivo.

Notes: (A) Application in anticancer treatment with intratumoral (AuNP embedded) liposome delivery and optical fiber guided laser excitation. Fluorescein was encapsulated in liposome for simulating the drug content release profiles. (B) Xenograft mice model experiment with fiber-optic conveyed laser excitation inside isoflurane chamber. (C) A computed tomography (fluoroscopy) guided needle insertion (similar or smaller needle size to percutaneous biopsy of thoracic spine tumor as figure shown) could be applied to place the device's fiber bundle probe to reach deeper tumor.

Abbreviation: AuNP, gold nanoparticle.

responsive liposomes for topical release in vivo (in tumors of xenograft mice, Figure 1B) using a $200 \mu \mathrm{m}$ optical fiber and a $65 \mathrm{~mW}$ continuous wave (CW) diode laser (Figure 1A). The photo-thermally reactive liposomes consisted of hydrophobic gold nanoparticles (AuNPs) tethered to the membrane of a temperature-responsive liposome; this design was responsible for converting light energy into heat by surface plasma resonance to increase liposome permeability when reaching the phase transition temperature. ${ }^{16,21,31}$ AuNPs are well recognized as biocompatible and have been applied in medical studies for decades, and thermal responsive liposomes are under clinical trials, such as 1,2-dipalmitoyl-sn-glycero-3-phosphatidylcholine (DPPC)-based Thermodox in Phase III. ${ }^{32,33}$ The pattern of triggered content release was quantified by an increase in fluorescence (in vivo fluorescence imaging) due to the dilution of a $75 \mathrm{mM}$ (self-quench concentration) fluorescein solution when released from the liposome (Figure 2).$^{34}$

\section{Experimental Materials}

All reagents and solvents were acquired from SigmaAldrich (St Louis, MO, USA) and used as received unless otherwise indicated. DPPC, 1-myristoyl-2-palmitoylsn-glycero-3-phosphotydilcholine (MPPC), 1,2-distearoyl-sn-glycero-3-phosphatidylcholine (DSPC), and 1,2-distearoyl-sn-glycero-3-phosphoethanolamine-N[methoxy (polyethylene glycol)-2000] (DSPE-PEG2k) were obtained from Avanti Polar Lipids (Alabaster, AL, USA) stored at $-20^{\circ} \mathrm{C}$ in chloroform. Sephadex G-75 was obtained from GE Healthcare Life Science (Pittsburgh, PA, USA) and hydrated in deionized water at $4^{\circ} \mathrm{C}$ overnight before applied for gel filtration.

\section{Synthesis and characterization of AuNP}

The synthesis method was adapted from literature by singlephase reduction of gold trichloride. ${ }^{35}$ In brief, $7.5 \mathrm{mg} \mathrm{AuCl}{ }_{3}$ dissolved in $1 \mathrm{~mL}$ didodecyldimethylammonium bromide was reduced by $25 \mathrm{mg}$ tetrabutylammonium borohydride dissolved in $1 \mathrm{~mL}$ didodecyldimethylammonium bromide solution in the presence of $43 \mathrm{mg}$ decanoic acid dissolved in $2.5 \mathrm{~mL}$ toluene under vigorous mixing overnight, then stabilized by adding $79 \mu \mathrm{L} 0.5 \mathrm{M}$ tri-n-octylphosphine in toluene at $60^{\circ} \mathrm{C}$ for 5 minutes. After cooling to room temperature, the AuNPs were recrystalized by dropwise addition of $300 \mu \mathrm{L}$ 

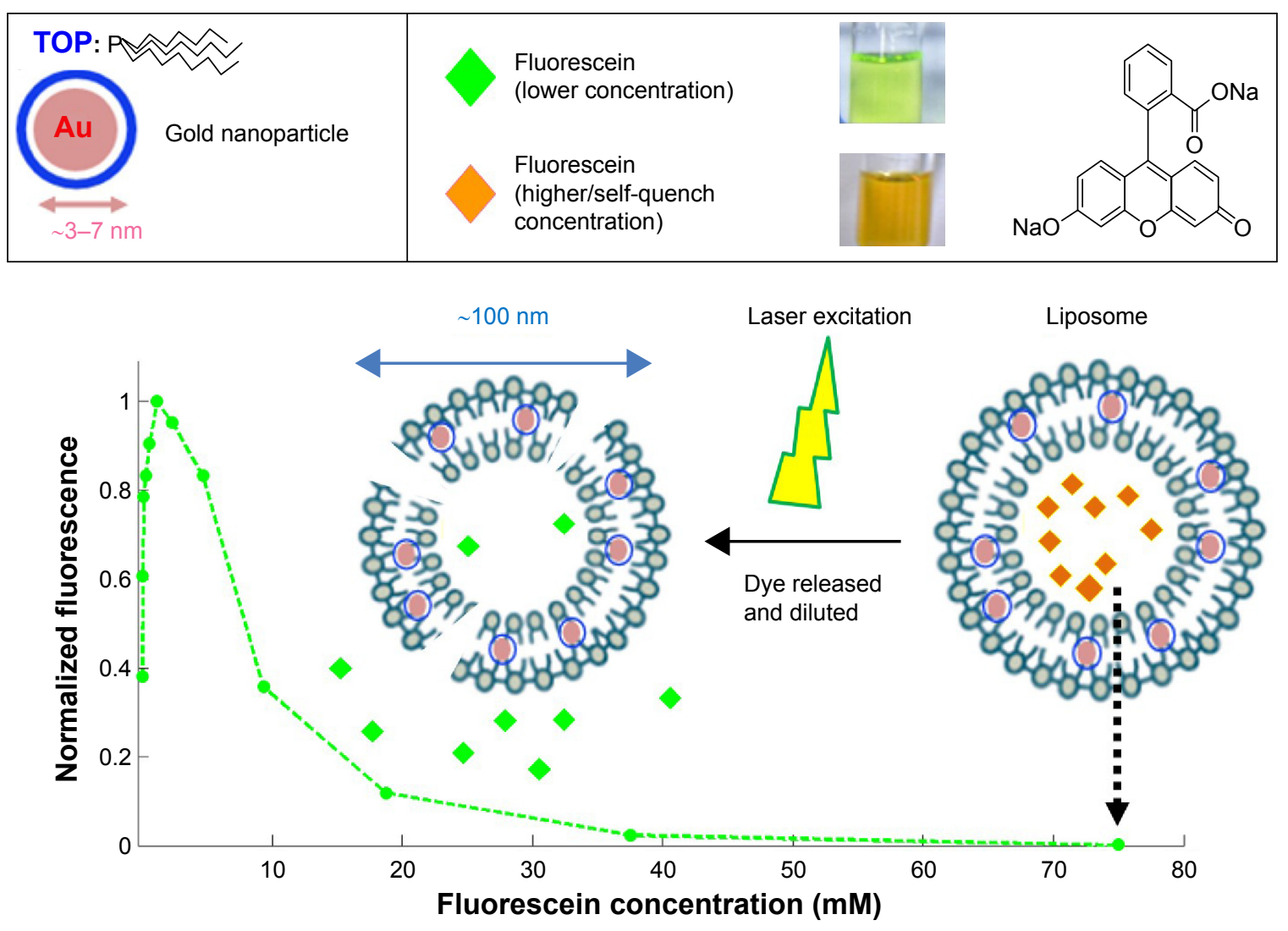

Figure 2 Quantification of liposomes release by mitigation of fluorescein self-quench.

Notes: Fluorescein solution in self-quench concentration $(75 \mathrm{mM})$ was encapsulated in liposome. After fiber-optic triggered photo-thermal responsive release, discharged fluorescein from liposome was diluted and emitted brighter fluorescence.

Abbreviation: TOP, tri-n-octylphosphine.

methanol and separated by centrifugation at 3,000 rpm for 5 minutes. The precipitated AuNPs were then dissolved in chloroform and characterized by absorption spectrum (Synergy ${ }^{\mathrm{TM}}$ Mx Multi-Mode Microplate Reader, Biotek, Winooski, VT, USA) with $1 \mathrm{~cm}$ pathlength Bio-Cell ${ }^{\mathrm{TM}}$ for confirmation of AuNPs existence with the surface plasma resonance peak. Particle size distribution of the AuNP/chloroform solutions was measured by dynamic laser scattering (DLS, Zetasizer Nano, Malvern Instruments, Malven, UK). AuNPs were deposited onto a carbon coated copper grid after evaporation of chloroform in air and inspected by high-resolution transmission electron microscopy (HR-TEM, JEM-2010, Jeol, Tokyo, Japan) with selected particle diameter calculated by Image J software (1.48v, NIH, Bethesda, MD, USA) when compared with scale bar on the images. AuNPs were deposited onto a glass slide after evaporation of chloroform in air and inspected by X-ray diffractometer (Bruker, Billerica, MA, USA) for lattice structure identification. Concentration of AuNP/chloroform solutions was determined by first estimating the extinction coefficient $(\varepsilon)$ from the average core diameter (D) of AuNPs measured by HR-TEM with the formula: $\ln \varepsilon=3.32 \ln \mathrm{D}+10.8$, and then calculated the concentration from the absorbance of surface plasma resonance peak (A) with the formula of Beer-Lambert law: $\mathrm{A}=\varepsilon \times 1$ (pathlength of Bio-Cell ${ }^{\mathrm{TM}}$ in $\left.\mathrm{cm}\right) \times \mathrm{C}$ (concentration in $\mathrm{M})$ as literature. ${ }^{36}$

\section{Photo-thermal conversion of AuNP by fiber-optic guided laser excitation}

In all, $2 \mathrm{~mL}$ AuNPs solution in chloroform at different concentrations was loaded in $15 \times 125 \mathrm{~mm}$ pyrex test tubes with silicone seal. The solution in each test tube was wrapped with aluminum foil and stimulated with $200 \mu$ m multi-mode optical fiber (FIBER-200-VIS, OceanOptics, Dunedin, FL, USA) guided $65 \mathrm{~mW}$ diode pumped solid-state laser (TWC Opto Corp, Taipei, Taiwan) with the emission end of the optical fiber was placed beneath the surface of the solution through the silicone seal and $2 \mathrm{~cm}$ above the bottom of the test tube (Figure 3A). A digital thermometer (GE-363D, Dr AV Technology Co. Ltd., Changhua, Taiwan) was also placed beneath the surface of the solution through the silicone seal about the same depth of the end of optical fiber to monitor 


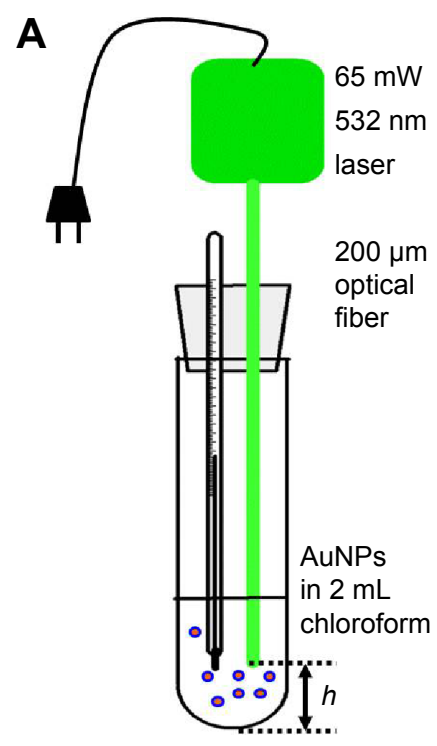

B
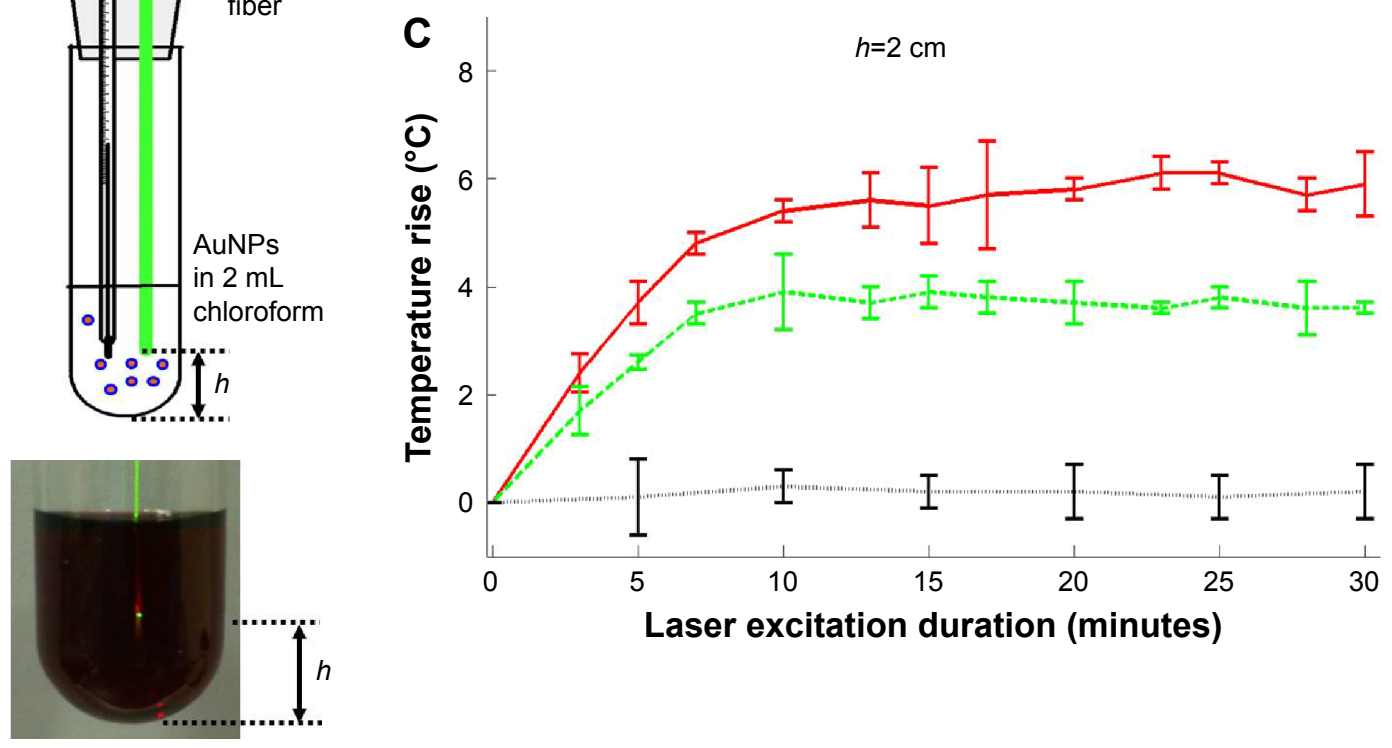

Figure 3 Photo-thermal energy conversion by AuNPs in chloroform.

Notes: (A) Illustration of apparatus. (B) Appearance of collimated laser beam exiting optical fiber and illuminating into dark-reddish AuNP solution in chloroform. (C) AuNPs concentration and excitation duration dependent photo-thermal conversion profiles with fixed light path $(h=2 \mathrm{~cm})$. Data of $(\mathbf{C})$ represent mean \pm standard deviation $(\mathrm{n}=3)$. Abbreviation: AuNP, gold nanoparticle.

the excitation time-dependent temperature changes of the solution under continuously excitation.

\section{Fabrication and characterization of photo-thermal responsive liposome}

The fabrication method was adapted from the literature by the lipid film hydration extrusion method. ${ }^{37}$ In brief, phospholipids or cholesterol (Chol) in chloroform were mixed in molar ratios 90:10:4 (DPPC:MPPC:DSPE-PEG2k) or 90:10 (DSPC:DPPC), or 80:20 (DPPC:Chol) for achieving a lipid concentration of $20 \mathrm{mg} / \mathrm{mL}$ with AuNP/chloroform concentrations ranged $0-132 \mathrm{ng} / \mathrm{mL}$, dried into a thin film by convection of nitrogen and followed by vacuum overnight. The dried lipid/AuNPs film was rehydrated with isotonic $75 \mathrm{mM}$ fluorescein solution under sonication at $55^{\circ} \mathrm{C}$. The resultant liposomes were extruded gradually through 400 , 200 , and $100 \mathrm{~nm}$ polycarbonate membranes (21 times each) to form large unilamellar vesicles. ${ }^{38}$ The unencapsulated fluorescein was then removed by column filtration with Sephadex G-75 (GE Healthcare, Pittsburgh, PA, USA) applying isotonic saline as elute phase, and collected with lipid concentration at $5 \mathrm{mg} / \mathrm{mL}$ measured by phospholipid assay (Wako Chemicals USA, Inc, Richmond, VA, USA). The mean diameter of the liposomes was verified by DLS (Zetasizer Nano, Malvern Instruments) and TEM (JEM-1400, Jeol). In isotonic saline solutions, the liposomes were found to maintain their structural stability for up to 5 weeks when stored at $4{ }^{\circ} \mathrm{C}$ with less than $0.1 \%$ payload leaking observed by fluorescence intensity when encapsulation with $75 \mathrm{mM}$ (self-quench) fluorescein solution.

\section{Fiber-optic guided laser excitation triggered liposome release in vitro}

In all, $2 \mathrm{~mL} 75 \mathrm{mM}$ fluorescein entrapped liposome solution with a lipid concentration of $5 \mathrm{mg} / \mathrm{mL}$ was loaded in a $15 \times 125 \mathrm{~mm}$ pyrex test tube with silicone seal, wrapped with aluminum foil and pre-warmed to $37^{\circ} \mathrm{C}$ by immersing in water bath. The solution was stimulated with $200 \mu \mathrm{m}$ multi-mode optical fiber (FIBER-200-VIS, OceanOptics) guided $65 \mathrm{~mW}$ diode pumped solid-state laser (TWC Opto Corp, Taoyuan, Taiwan) with the emission end of the optical fiber was placed beneath the surface of the solution and $2 \mathrm{~cm}$ above the bottom of the test tube (Figure 4A). The fibercoupled laser module had $\mathrm{CW}$ emission beam as circular spot 
with $<5 \%$ power fluctuation, $<0.5 \mathrm{~nm}$ spectral line-width and coupled into optical fiber with SubMiniature version A type connector. The self-quenching property of fluorescein at a concentration of $75 \mathrm{mM}$ was used to quantify the percent fluorophore release from liposome. Fluorescein fluorescence is self-quenched inside the liposomes, but the fluorescence quenching is relieved after its release from liposome (Figure 2). ${ }^{34}$ Aliquots of $50 \mu \mathrm{L}$ liposome solution were sampled at different intervals during laser excitation, then mixed with $50 \mu \mathrm{L}$ isotonic saline, loaded in wells of 96-well plate, and their fluorescent emission intensities were measured by end-point detection of microplate reader (Synergy ${ }^{\mathrm{TM}} \mathrm{Mx}$ Multi-Mode Microplate Reader, Biotek) applying $488 \mathrm{~nm}$ as an excitation wavelength and $520 \mathrm{~nm}$ as an emission wavelength. The percent fluorophore release was determined by the following formula: $\left[\left(I_{t}-I_{i}\right) /\left(I_{d}-I_{i}\right)\right] \times 100 \%$, where $I_{t}$ was the measured intensity at time $t, I_{d}$ was the signal measured after liposome destruction by mixing aliquots of $50 \mu \mathrm{L}$ liposome solution with $50 \mu \mathrm{L}$ detergent (30 mM n-octyl- $\beta$-D-glucopyranoside), and $I_{i}$ was the measured initial fluorescence. Consecutive triggered release of the AuNP-liposomes was tested in saline or rat plasma. Rat plasma was collected from tail vein

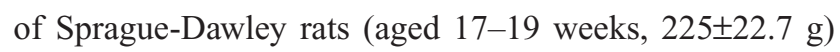
purchased from BioLASCO Taiwan Co, LTD (Yilan, Taiwan) after allowed to clot for a minimum of 45 minutes, and centrifugation at 2,000 rpm for 10 minutes to separate blood cells approved by the Institution Animal Care and Use Committee (IACUC) of National Chung Hsing University (IACUC Approval No 101-109).

\section{Murine xenograft model}

$\mathrm{Nu} /$ nu nude mice (aged 7-9 weeks, 32.5 $\pm 2.7 \mathrm{~g}$ ), purchased from BioLASCO Taiwan Co, LTD, were maintained in Experimental Animal Center of National Chung Hsing University, and studied following procedures approved by the IACUC of National Chung Hsing University (IACUC Approval No 101-108). Two to three mice were housed to each cage in an individually ventilated, temperature
A

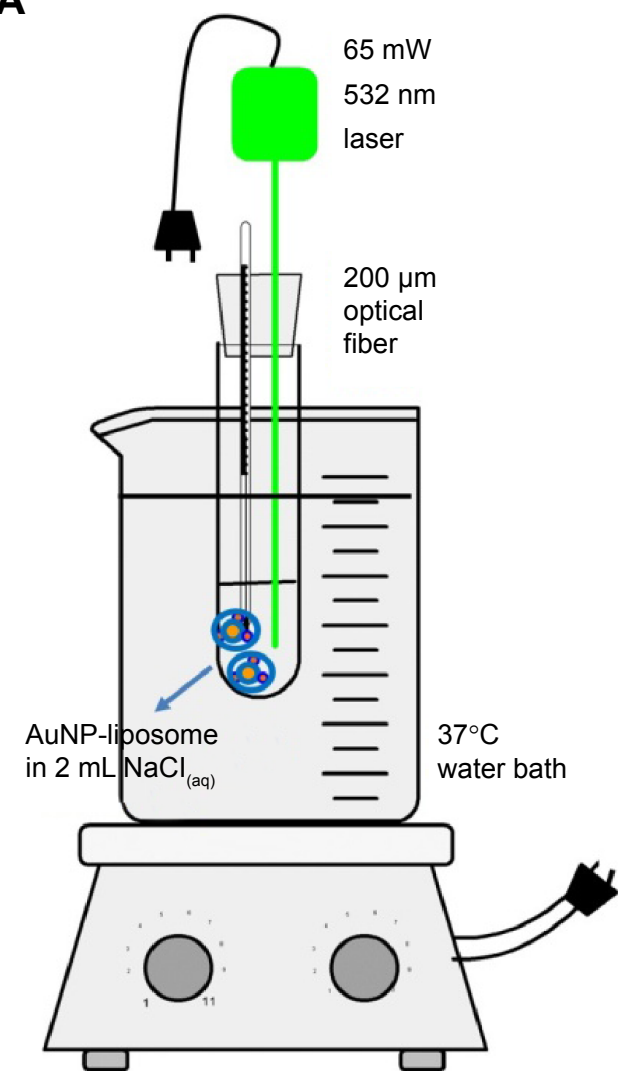

B

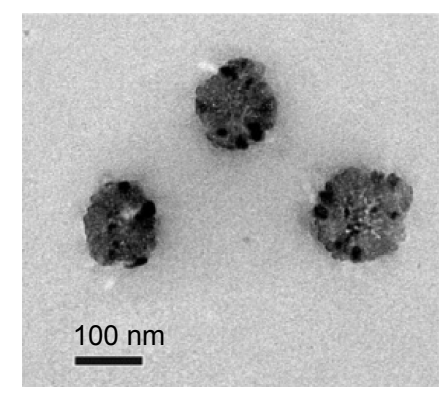

C

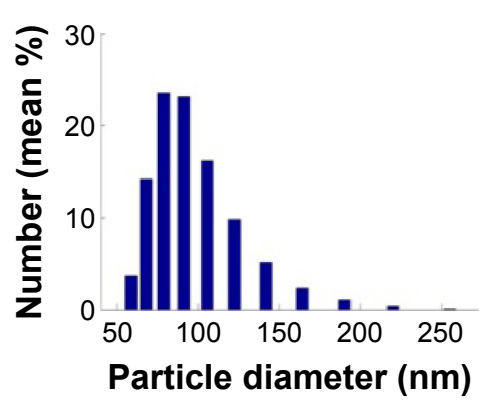

D

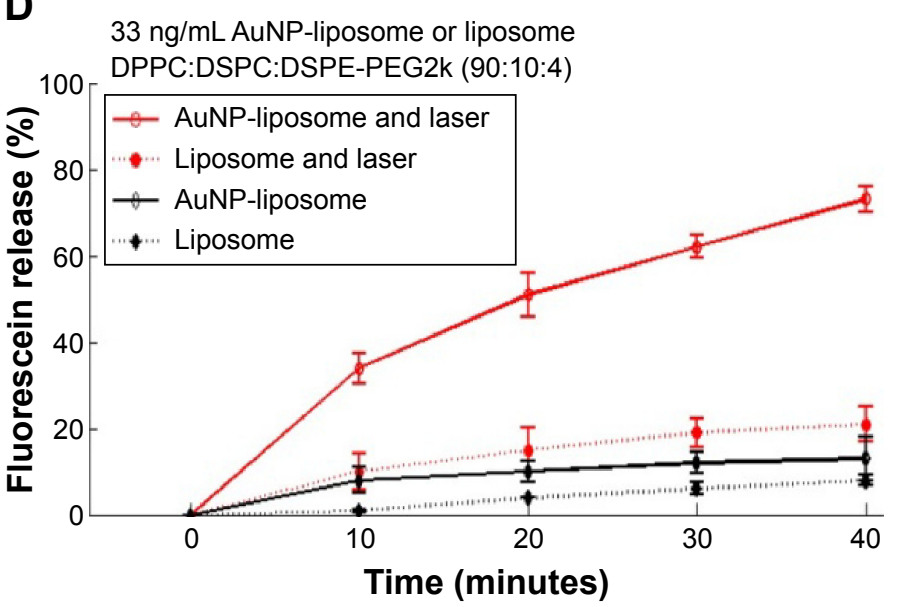

Figure 4 Fiber-optic guided laser excitation triggered photo-thermal responsive liposomes release in vitro.

Notes: (A) Illustration of apparatus. (B) Appearance of AuNP-liposome observed by transmission electron microscopy. (C) Particle size distribution of AuNP-liposome detected by dynamic laser scattering. (D) Contribution of AuNPs in photo-thermal responsive release of liposome. Data of (D) represent mean \pm standard deviation ( $n=3$ ). Abbreviations: AuNP, gold nanoparticle; DPPC, I,2-dipalmitoyl-sn-glycero-3-phosphatidylcholine; DSPC, I,2-distearoyl-sn-glycero-3-phosphatidylcholine; DSPE-PEG2k, I,2-distearoyl-sn-glycero-3-phosphoethanolamine-N-[methoxy (polyethylene glycol)-2000]. 
$\left(23^{\circ} \mathrm{C} \pm 2^{\circ} \mathrm{C}\right)$, and humidity $(50 \%-55 \%)$ controlled facilities, on 12 hours light, 12 hours dark cycle, and had free access to sterilized laboratory chow and water. The human breast adenocarcinoma cell line, MDA-MB 231, was obtained from the National Health Research Institute Cell Bank (Hsinchu, Taiwan) and cultured as recommended by the American Type Culture Collection (Manassas, VA, USA) with culture reagents obtained from Quantum Biotechnology (distributor of Life Technologies, Inc. and Invitrogen, Taichung, Taiwan) unless otherwise indicated. The cell culture medium was Dulbecco Modified Eagle Medium (DMEM) with 10\% fetal bovine serine (FBS). Approximately $1 \mathrm{~mL}$ mixtures (volume ratio $=1: 1$ ) of phenol red-free matrigel matrix (Bertec Enterprise Co Ltd, distributor of BD Bioscience, Taichung, Taiwan) and culture medium (DMEM with 10\% FBS) with the suspension of $1 \times 10^{7}$ MDA-MB 231 cells were injected subcutaneously into both dorsal flanks (the same level above the dorsal-ventral adjunction) of the mice to grow xenografts with minimal widths around $1 \mathrm{~cm}$. The xenografts were allowed to develop for 5 weeks before characterization with in vivo imaging inspections.

\section{Fluorescent imaging in vivo}

The in vivo fluorescence images of the mice were acquired using a Xenogen IVIS ${ }^{\circledR}$ Imaging System 200 Series (Caliper Life Science/Xenogen, Alameda, CA, USA), which features a temperature-controlled, light-tight imaging chamber and a cryogenically cooled back-illuminated digital charge-coupled device camera. Before and during the examination periods, anesthesia of the mice was initiated and maintained using the inhalation of isoflurane in oxygen $(1 \%-2.5 \%$ isoflurane $)$ in the isolated chamber of the system. In all, $50 \mu \mathrm{L}$ liposome solution prepared as mentioned above (section "Fabrication and characterization of photo-thermal responsive liposome") was administrated directly into the center of tumors. Image acquisition and processing was accomplished with Living Image ${ }^{\circledR}$ Software V2.6 (Caliper Life Science/Xenogen) on a PCbased system. The mice were brought to the imaging chamber at several time points after dye injection, placed in the center of the field of view (deliberately chosen as $13 \mathrm{~cm} \times 13 \mathrm{~cm}$ ) with the aid of the green laser alignment projector grid, and scanned with an excitation passband at 445-490 nm, an emission passband at 515-575 nm, 3 seconds exposure time, medium binning, and 4 seconds f/stop. Both delineation of tumor boundary and calculation of normalized fluorescence at the tumor area on the in vivo fluorescent images were processed by programs coded in MATLAB R2013a software (The MathWork Inc, Natick, MA, USA).

\section{Results Characterization of AuNP}

The absorbance spectrum of the resultant dark-reddish chloroform solution (Figure 3B) had a well-defined surface plasma resonance band with a maximal absorbance at approximately $522 \mathrm{~nm}$ (Figure 5C), confirming the existence of AuNPs in the chloroform solution. Those hydrophobic AuNPs were spherical in shape and had a uniform particle size distribution around 3-7 nm, as observed with HR-TEM (Figure 5A). Further examination by DLS analysis suggested that the particle size ranged from $2 \mathrm{~nm}$ to $11 \mathrm{~nm}$, mainly distributed from $3 \mathrm{~nm}$ to $7 \mathrm{~nm}$ with an average value of $5.55 \pm 2.1 \mathrm{~nm}$, and had size distribution polydiversity index smaller than 0.079 (Figure 5B). The crystal lattice of the nanostructure was in face-centered cubic shape, as identified by the X-ray diffraction pattern with several lattice planes ( $\left.\begin{array}{lll}1 & 1 & 1\end{array}\right),\left(\begin{array}{lll}2 & 0 & 0\end{array}\right)$,

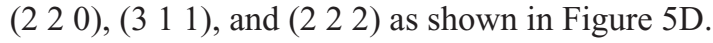

\section{Photo-thermal energy conversion efficiency of AuNPs in vitro}

To investigate the photo-thermal energy conversion efficiency of AuNPs, a fiber-optic guided $65 \mathrm{~mW}$ laser emitting at $532 \mathrm{~nm}$ (near the surface plasma resonance band of AuNPs at approximately $522 \mathrm{~nm}$, Figure 5C) was used to stimulate $2 \mathrm{~mL}$ AuNPs in chloroform at several concentrations. The laser, upon exiting the internal end (beneath the surface of the solution) of the optical fiber, propagated as a collimating beam, penetrating the dark-reddish AuNP/chloroform solution at all concentrations examined in the study, and formed a circular spot on the bottom surface of the test tube as shown in Figure 3B. The temperature increases of the AuNP/chloroform solutions after laser stimulation were recorded at regular time intervals to indicate the efficiency of conversion. It was found that the photo-thermal conversion efficiency (temperature rise) was in proportion to the AuNP concentration and excitation duration when excitation light path $(h)$ was fixed at $2 \mathrm{~cm}$ before reaching a plateau approximately 7-10 minutes after laser stimulation (Figure 3C). The excitation band of the green laser (532 nm) is far from the absorption band of the solvent, chloroform (at $<250 \mathrm{~nm}$ ), which was reflected in the relatively insignificant temperature rise $\left(0.2^{\circ} \mathrm{C} \pm 0.51^{\circ} \mathrm{C}\right)$ of a solution without AuNPs. With AuNPs at $4.5 \times 10^{-7}$ or $9 \times 10^{-7} \mathrm{M}$, the solutions were heated at $3.9^{\circ} \mathrm{C} \pm 0.15^{\circ} \mathrm{C}$ or $5.9^{\circ} \mathrm{C} \pm 0.63^{\circ} \mathrm{C}$, respectively.

\section{Fiber-optic guided laser triggered liposome release in vitro}

Three of the most common thermal responsive liposomes formula, 90:10:4 (DPPC:MPPC:DSPE-PEG2k) or 90:10 
A

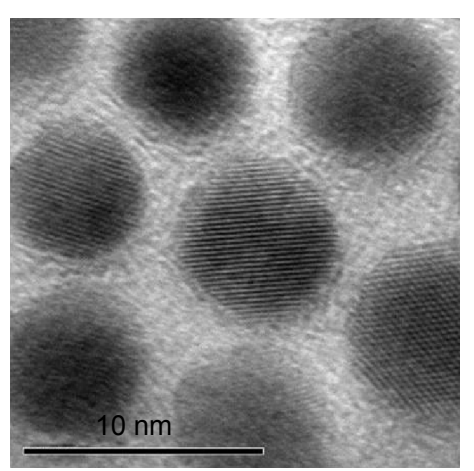

\section{C}

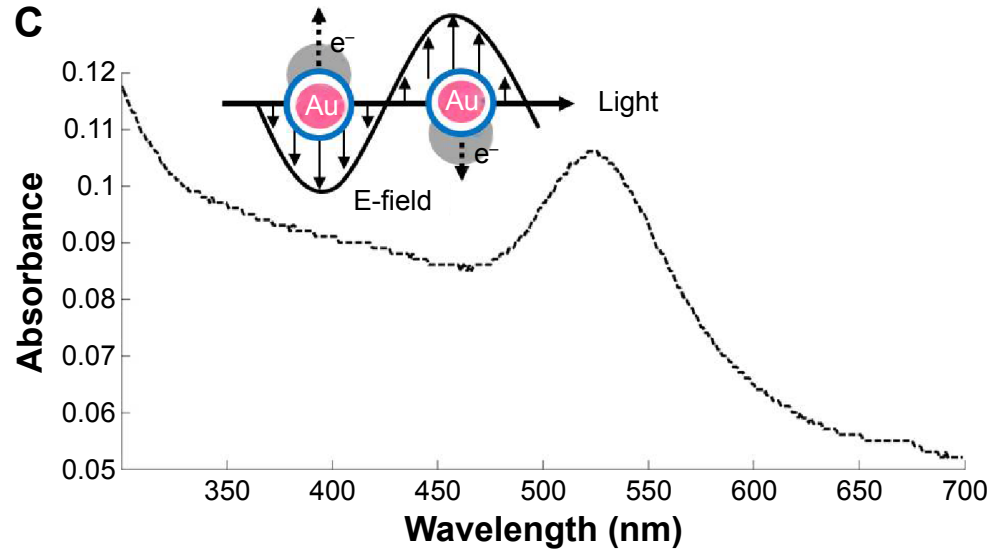

B

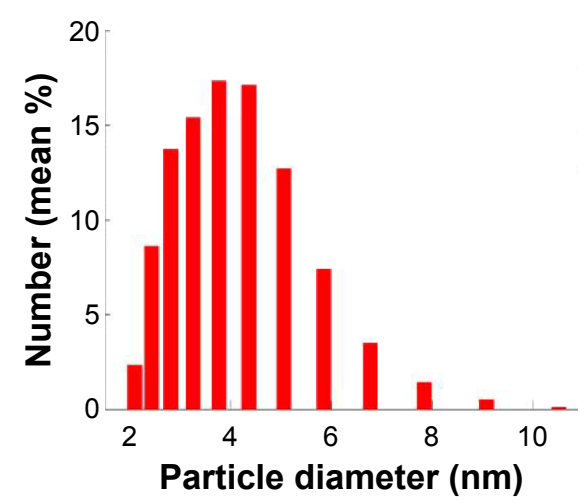

D

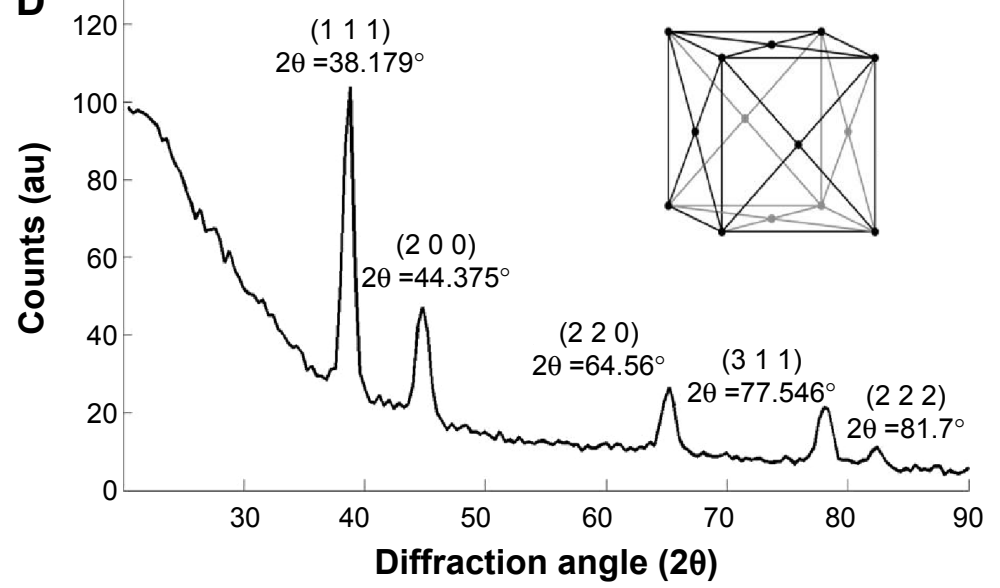

Figure 5 Characterization of synthesized gold nanoparticles.

Notes: (A) Morphology observed by transmission electron microscopy (black bar $=10 \mathrm{~nm}$ ). (B) Particle size distribution detected by dynamic laser scattering. (C) Surface plasma resonance band identified by absorbance spectrum with a maximal absorbance at approximately $522 \mathrm{~nm}$. (D) Face-centered crystal lattice indicated by X-ray diffraction spectrum.

Abbreviation: au, arbitrary unit.

(DSPC:DPPC), or 80:20 (DPPC:Chol), were tethered with AuNPs up to $132 \mathrm{ng} / \mathrm{mL}$ for exploring the optimal photoresponsive liposome for the fiber-optic guided laser triggered release study. ${ }^{17,26,27,39-42}$ The 90:10:4 (DPPC:MPPC:DSPEPEG2k) liposome with $33 \mathrm{ng} / \mathrm{mL}$ AuNPs achieved the strongest photo-thermal conversion efficiency while maintaining decent liposome integrity at physiological temperature without causing significant leakage $(21.92 \% \pm 2.08 \%$ in 40 minutes without excitation) as shown in Figure 6. The AuNP-incorporated liposomes were spherical in shape with AuNPs appearing as black dots floating on the surface, and had a uniform particle size distribution, primarily around $100 \mathrm{~nm}$, as observed by TEM (Figure 4B). Further examination with DLS analysis suggested that the particle size ranged primarily from $78 \mathrm{~nm}$ to $122 \mathrm{~nm}$ with an average value of $91.28 \pm 12.1 \mathrm{~nm}$ (Figure 4C), and had size distribution polydiversity index smaller than 0.121. The embedment of AuNPs in the liposome membrane under laser excitation significantly enhanced the permeability of the liposome and triggered more efficient release $(74.53 \% \pm 1.63 \%$ in 40 minutes, red solid curve) than liposomes without AuNPs $(14.53 \% \pm 3.17 \%$ in 40 minutes, black solid curve) or AuNP-liposomes without excitation $\left(21.92 \% \pm 2.08 \%\right.$ in 40 minutes, red dot curve) in vitro $\left(37^{\circ} \mathrm{C}\right.$ water bath) as shown in Figure 4D.

\section{Effect of liposome encapsulation on diffusion of fluorophores in tumor}

Malignant xenografts (MDA-MB 231 cell/matrigel ${ }^{\mathrm{TM}}$ ) were implanted subcutaneously into male nu/nu nude mice and allowed to grow intoa solidmass with diameterfrom $1 \mathrm{~cm}$ to $2 \mathrm{~cm}$. After anesthesia, the mice received a $50 \mu \mathrm{L}$ bolus injection of either $1 \mathrm{mM}$ fluorescein aqueous solution or $1 \mathrm{mM}$ fluorescein encapsulated in AuNP-liposomes (the equivalent fluorescein concentration was $0.25 \mathrm{mM}$ due to the dilution effect after purification with size-exclusive gel filtration to remove unencapsulated fluorescein; the lipid concentration was similarly diluted from $20 \mathrm{mg} / \mathrm{mL}$ after hydration to $5 \mathrm{mg} / \mathrm{mL}$ after purification). A $1 \mathrm{mM}$ fluorescein aqueous solution was 

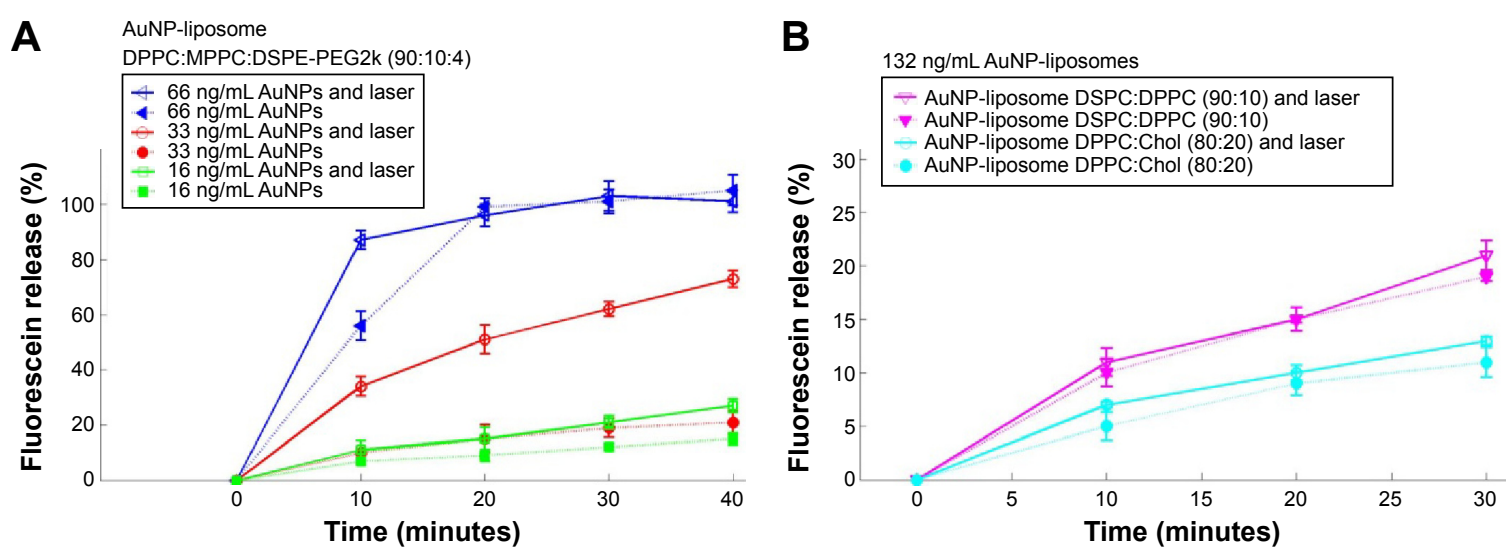

Figure 6 Optimal composition of photo-thermal liposome.

Notes: (A) Effect of AuNP concentration. DPPC:MPPC:DSPE-PEG2k (90:10:4) liposome with $33 \mathrm{ng} / \mathrm{mL}$ AuNP had photo-thermal conversion efficiency and stability among AuNP concentrations from $16 \mathrm{ng} / \mathrm{mL}$ to $66 \mathrm{ng} / \mathrm{mL}$. (B) Effect of phospholipid compositions. Both DSPC:DPPC (90:10) and DPPC:Chol (80:20) liposomes had insufficient photo-thermal conversion efficiency and stability.

Abbreviations: AuNP, gold nanoparticle; Chol, cholesterol; DPPC, I,2-dipalmitoyl-sn-glycero-3-phosphatidylcholine; DSPC, I,2-distearoyl-sn-glycero-3-phosphatidylcholine; DSPE-PEG2k, I,2-distearoyl-sn-glycero-3-phosphoethanolamine-N-[methoxy (polyethylene glycol)-2000]; MPPC, I-myristoyl-2-palmitoyl-sn-glycero-3-phosphotydilcholine.

chosen because it had the highest concentration-dependent quantum yield, providing the strongest signal in studying the diffusion of fluorophores (within or without liposome) by fluorescent imaging. The time-dependent diffusion patterns of fluorophores (within or without liposome) were monitored by in vivo planar fluorescence imaging. The tumor boundary was delineated with specific fluorescence contour levels of pixels on bright-field (BF) images of mice at times after fluorophore (within or without liposome) injection, such as 0.5 at $0^{+}$minute and 30 minutes, 0.25 at 60 minutes, and 0.071 at 120 minutes for the $1 \mathrm{mM}$ fluorescein group (Figure $7 \mathrm{~A}$ ) or 0.5 at $0^{+}$minute and 30 minutes, 0.3 at 60 minutes and 120 minutes for the liposome group (Figure 7B). The normalized fluorescence at the tumor area was calculated by dividing the sum of the fluorescence intensities of the pixels in the tumor (above the threshold contour level) at
A
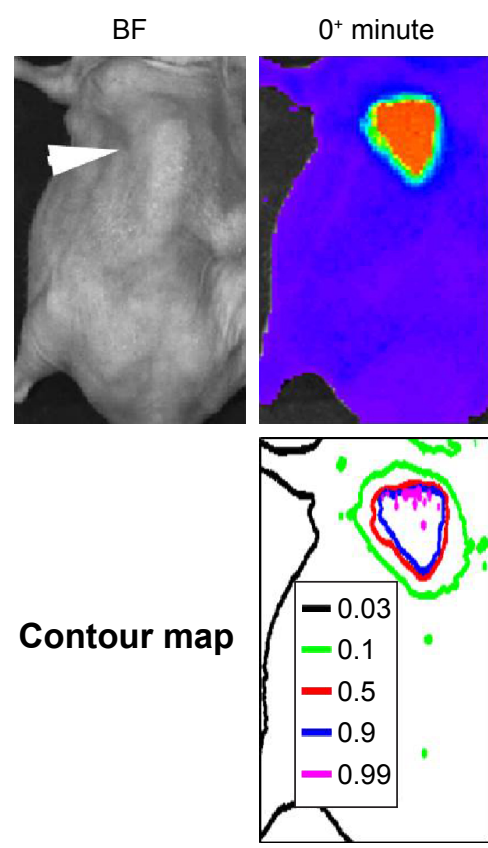

$1 \mathrm{mM}$ fluorescein
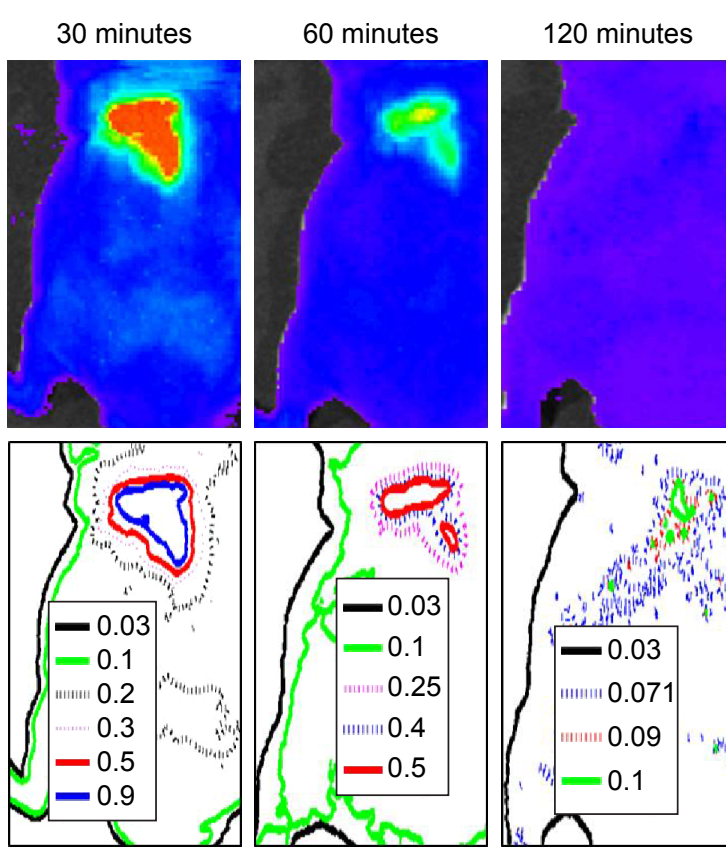

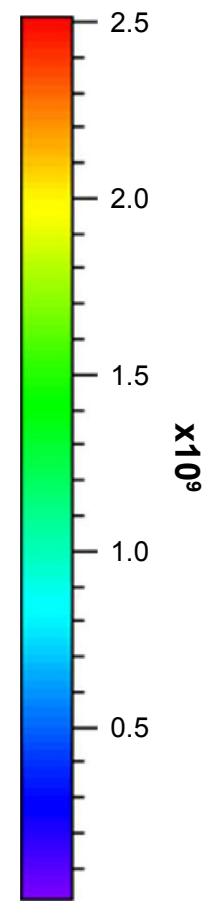

Figure 7 (Continued) 
B

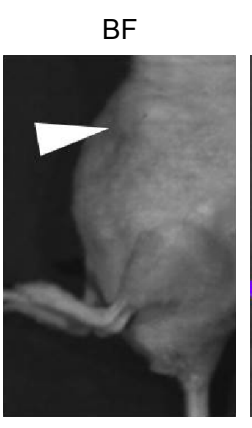

Contour map
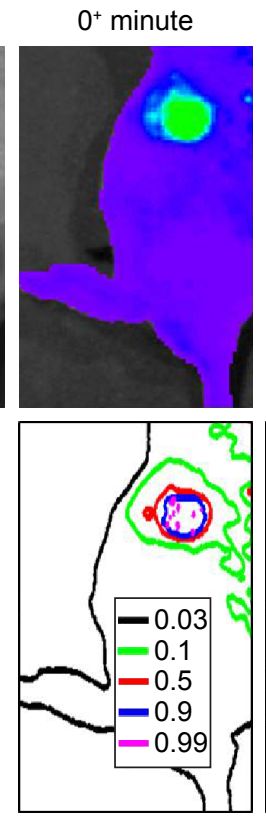

$1 \mathrm{mM}$ fluorescein in AuNP-liposome

30 minutes
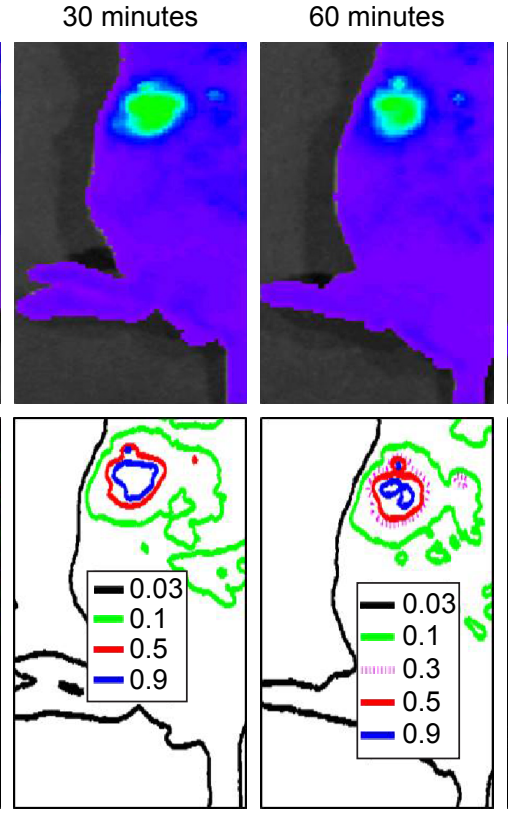
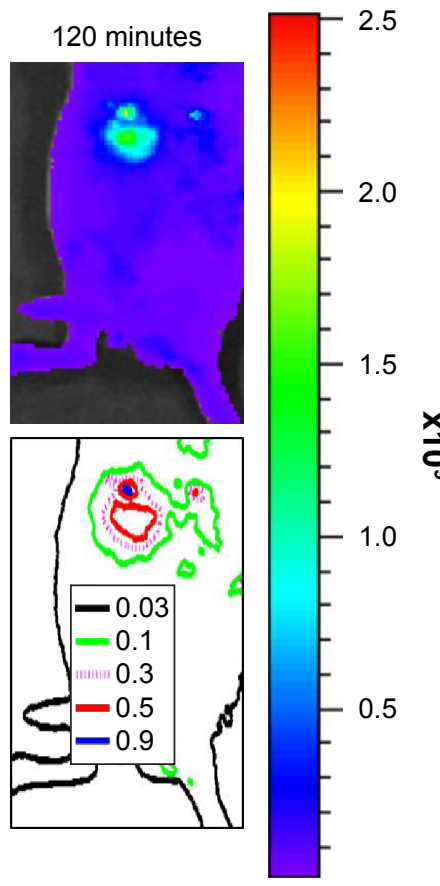

\section{$\underset{\overrightarrow{0}}{\stackrel{x}{0}}$}

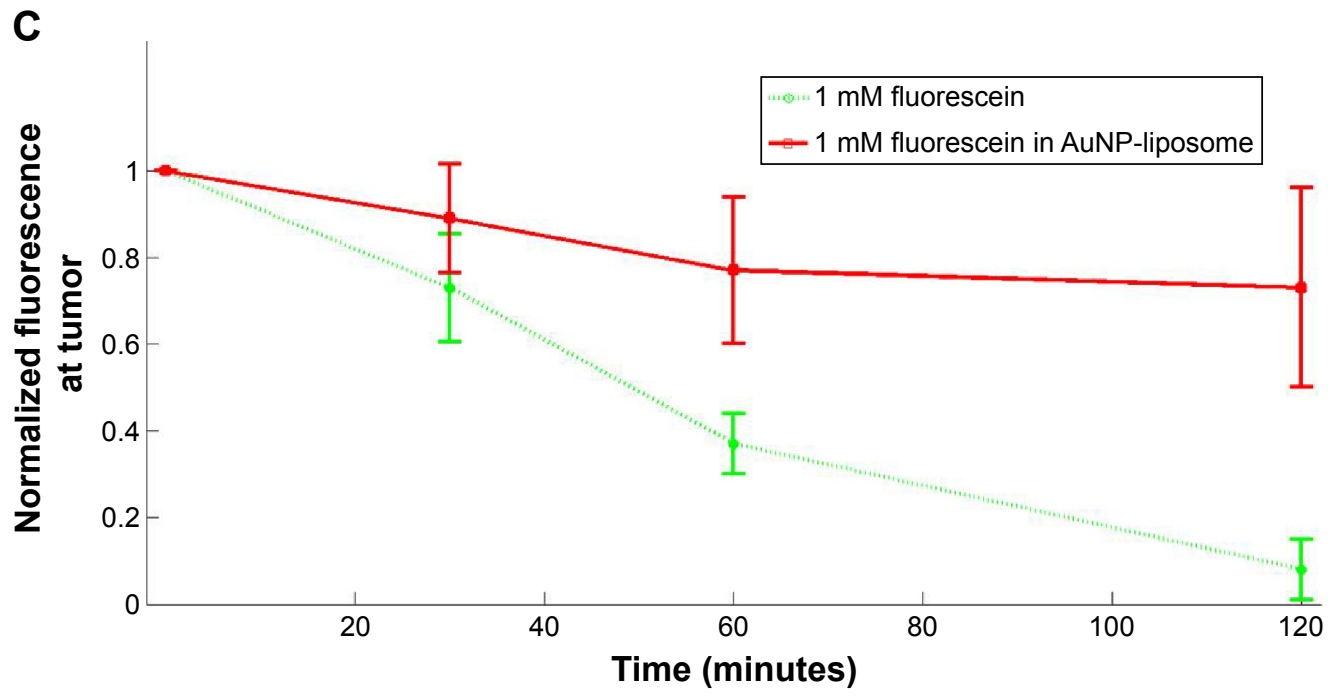

Figure 7 Effect of liposome encapsulation on diffusion of fluorophores in tumor.

Notes: (A) Diffusion profile of I mM free fluorescein in tumor. (B) Diffusion profile of I mM fluorescein encapsulated liposome in tumor. (C) Quantification of diffusion profiles. The positions of tumors in (A) and (B) are indicated by the white arrowhead in the bright-field (BF) images. Data of (C) represent mean \pm standard deviation ( $\mathrm{n}=5$ ). Abbreviation: AuNP, gold nanoparticle.

a specific time by the sum at $0^{+}$minute (Figure 7 C). It was found that liposome encapsulation yielded approximately $81 \%$ fluorophore retention (estimated by the normalized fluorescence intensity at the 120 th minute) in the tumor 120 minutes after fluorophore injection (Figure 7C, red curve), while less than $14 \%$ free fluorophore retention was observed in the tumor 120 minutes after fluorophore injection (Figure 7C, green curve).

\section{Fiber-optic guided laser excitation} triggered liposome release in vivo

Fiber-optic guided laser excitation triggered liposome releases in vivo were investigated with the xenograft mice model mentioned above. After anesthesia, the mice received a $50 \mu \mathrm{l}$ bolus injection of $75 \mathrm{mM}$ fluorescein aqueous solution encapsulated in AuNP-liposomes. A 10-minute duration of $\mathrm{CW}$ laser excitation was selected for consecutive tunable 
A

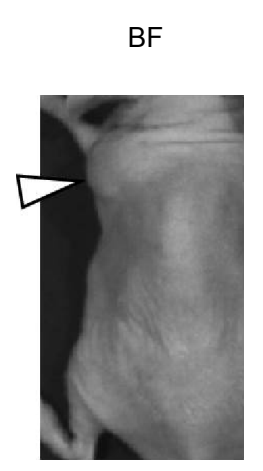

Contour map

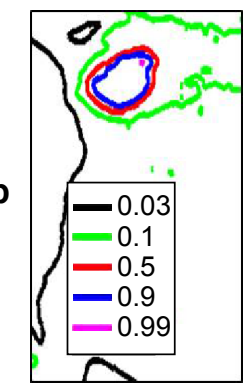

$$
\text { Before }
$$$$
\text { laser }
$$

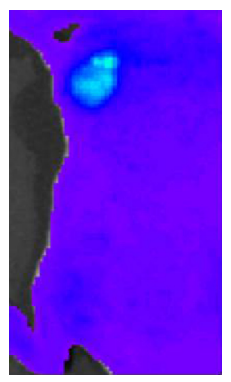

$75 \mathrm{mM}$ fluorescein in AuNP-liposome

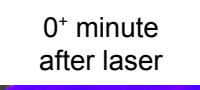

60 minutes
after laser
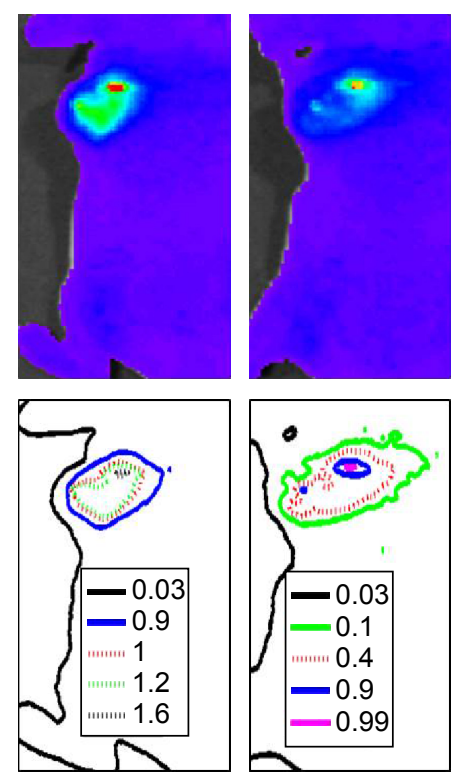
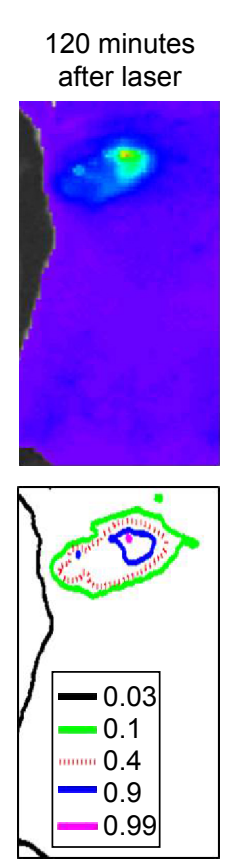

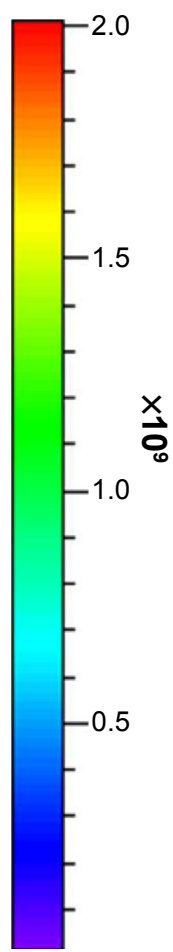

B

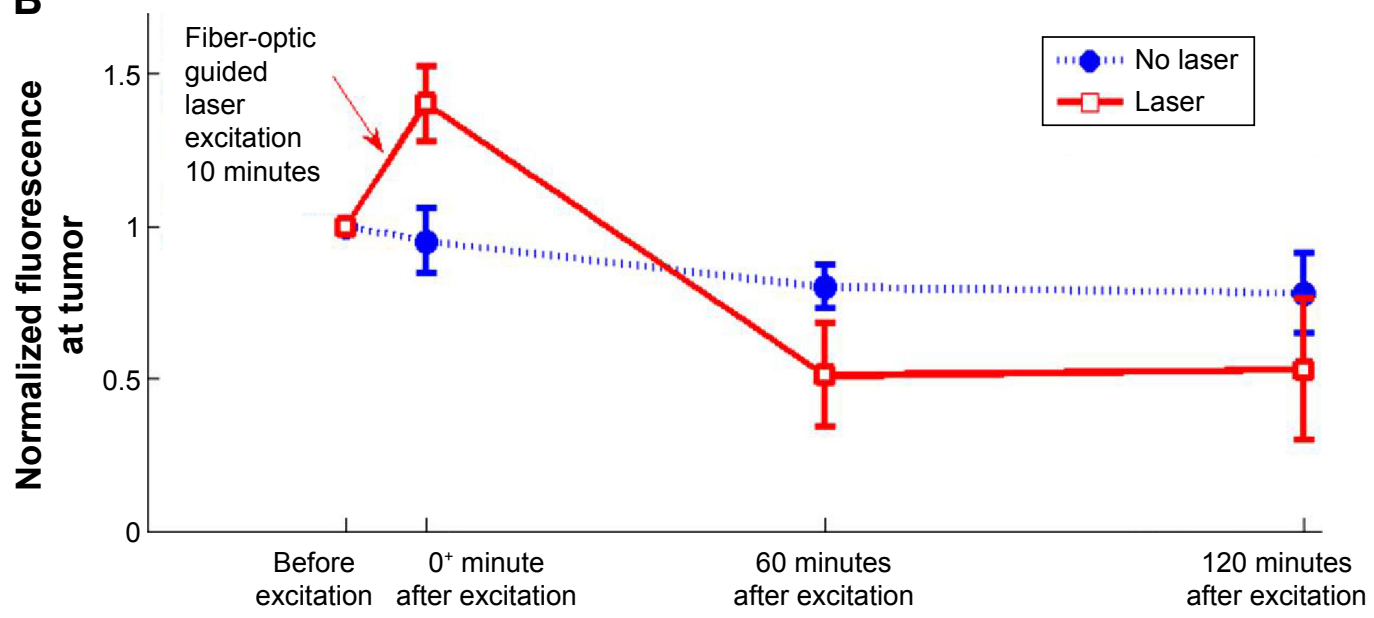

Figure 8 Fiber-optic guided laser excitation triggered photo-thermal responsive liposomes release in vivo.

Notes: (A) Releasing profile of $75 \mathrm{mM}$ fluorescein (in self-quench concentration) encapsulated in liposome with excitation. (B) Quantification of releasing profiles with or without excitation. The position of tumor in $(\mathbf{A})$ is indicated by the white arrowhead in the bright-field $(B F)$ images. Data of $(\mathbf{B})$ represent mean \pm standard deviation ( $\mathrm{n}=5$ ). Abbreviation: AuNP, gold nanoparticle.

release (with $\sim 50 \%$ fluorescein remaining in the liposome after the first triggered release, see Figure 4D, red curve). The tumor boundary was delineated with specific fluorescence contour levels of pixels on bright field images (BF) of mice at times after $75 \mathrm{mM}$ fluorescein in AuNp-liposome injection, such as 0.5 before laser excitation, and 1, 0.4, 0.4 at $0^{+}$, 60, $120 \mathrm{~min}$ (Figure 8A). The laser excited liposomes had a significant $\left(+42 \%\right.$, [normalized fluorescence $0^{+}$minute after excitation-normalized fluorescence before excitation]/normalized fluorescence before excitation) normalized fluorescence increase at the tumor area immediately after 10 minutes of excitation (Figure 8B, red curve), while liposome without excitation had gradually declined normalized fluorescence at the tumor area (Figure 8B, blue curve). 
A

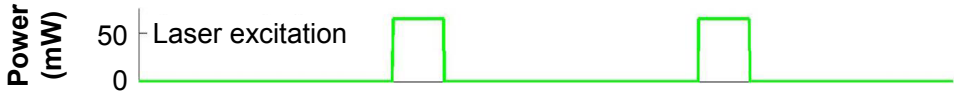

B

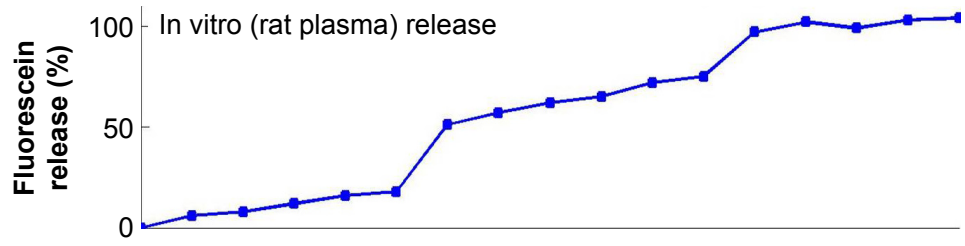

C

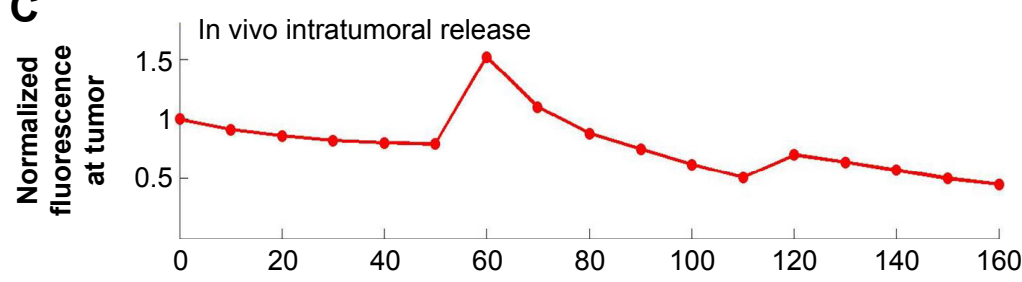

Figure 9 Profile of triggered liposome release under consecutive pulsatile excitation. Notes: (A) Laser excitation durations. (B) Consecutive release in vitro (rat plasma). (C) Consecutive release in vivo (mice xenograft).

\section{Potential of the delivery system for tunable release}

Upon consecutive fiber-optic guided laser excitation of $75 \mathrm{mM}$ liposome-encapsulated fluorescein in vivo, a significant (72\%, [normalized fluorescence at 60 th minute - normalized fluorescence at 50th minute]/normalized fluorescence at 50th minute) fluorescence increase was observed at the tumor area immediately after the first 10 minutes (50th-60th minutes, Figure 9A) of laser excitation, while a relatively milder fluorescence increase (33\%, [normalized fluorescence at 120th minute-normalized fluorescence at 110th minute]/ normalized fluorescence at 110th minute) was observed after the second 10 minutes (110th-120th minutes, Figure 9A) of laser excitation (Figure 9C), which was consistent with accumulation fluorescein quantity upon consecutive fiber-optic guided laser excitation of $75 \mathrm{mM}$ liposome-encapsulated fluorescein in vitro (rat plasma) as shown in Figure 9B.

\section{Discussion}

The fiber-optic guided laser excitation triggered content release from AuNP-embedded liposomes was characterized both in vitro and in vivo. The hair-size $(200 \mu \mathrm{m})$ optical fiber was found to be capable of triggering sufficient release when conveying $65 \mathrm{~mW} \mathrm{CW}$ diode laser excitation with time intervals of minutes, not only in biomimetic $37^{\circ} \mathrm{C}$ saline solutions but also in xenograft tumors.

This study demonstrated for the first time the triggered release of selective content from AuNP-embedded liposomes in vivo. Because of their proven biocompatibility and efficient photo-thermal conversion through surface plasma resonance, AuNPs (cluster, nanoshell) have previously been deposited on the liposome membrane outer surface, tethered to the liposome membrane, or entrapped within the liposome cavity for remotely triggered content release through wavelength-specific light excitation from the ultraviolet to near infrared region. ${ }^{39-42}$ However, no studies have reported their corresponding content release efficiencies triggered by light excitation in vivo. This may be due to challenges caused by the inconsistent results of in vivo non-invasive irradiation from varying subject/disease conditions (such as tumor size and location). ${ }^{30}$ Our preliminary results proved that the transmission of external energy (laser excitation) through thin fibers (replacing propagation through inhomogeneous tissue) can eliminate the limited penetration efficiency and subject-dependent inconsistency of the conventional noninvasive external trigger systems (which still require invasive chemotherapy administration by intravenous or intratumoral injection) mentioned above, ${ }^{17,18,25-27}$ while yielding standard errors of excitation triggered release (Figure 8B) in a range (defined by the percentage of the normalized signal) similar to that of diffusion errors (Figure 7C). Both standard errors originated primarily from deviations of diffusion caused by subject-dependent bio-medium conditions, and fiber-optic guided excitation did not contribute further fluctuations to triggered releasing profiles. The illumination covered the whole area of tumors with diameters up to $2 \mathrm{~cm}$ (Figure 1B). The tunable delivery was controlled by the duration of excitation (light exposure time) as shown in Figure 4D, but could be further regulated by modulating laser output power/amplitude (total light dosage) or excitation pattern (light delivery mode). 
This could be used to overcome deviations owing to subject/disease conditions (such tumor size and location); further bedside dosage fine-tuning during procedure could be provided by pairing with minimally invasive fiber-optic monitoring of drug-induced apoptosis activity function as mentioned above. ${ }^{22-24}$

The tunable delivery through liposome was also found superior than the conventional dropwise intravenous or intratumoral injection because of dosage retention and side effect reduction. From the in vivo fluorophore diffusion studies (Figure 7), encapsulation in liposomes enabled the retention of more than $80 \%$ of the injected fluorescein (5.79 times higher than fluorescein administered without liposomes) after 120 minutes. For the group with fluorescein administered without liposomes (Figure 7A), a significant amount of the simulated drug-biodistribution marker escaped from the tumor through leaky neovascular tumor-to-circulation transfer, unlike the payload encapsulated in liposomes, which had a bulkier volume and lower mobility (or a reduced transfer efficiency through neovascular pores). Up to a $10 \%$ normalized dosage (compared with the highest concentration accumulated in the tumor at $0^{+}$minute) was readily spread systematically through the circulation between 30 and 60 minutes after injection (Figure 7A), but not in the liposome group (Figure 7B). This result indicated that the intended burst discharge profiles being developed for most internal and external trigger systems could result in undesired lateral damage on exposed non-target tissue. The active release mechanism should be fine-tuned by the minimally invasive fiber-optic monitoring described above to steadily maintain the released therapeutic dosage (subject/patient dependent) for an extended period. In addition, the experimental tunable chemotherapy agent delivery platform delivers photo-thermally responsive liposomes directly into the tumor for more efficient tumor-specific accumulation and replaces conventional systematic intravenous administration, which is unreliably effective because of the subject-dependent differences in systematic elimination and lesion tissue absorption of therapeutic agents. ${ }^{4}$ This design could also circumvent the limited penetration of anticancer agents into tumor tissue by conventional intravenous administration (with or without delivery vesicles) due to the high interstitial pressure of cancerous tissues. ${ }^{16,19}$ These therapeutic improvements will be verified by future in vivo studies delivering chemotherapy agents in place of the fluorescein used in this research with optical imaging, such as photo-acoustic imaging, interrogating the time-dependent biodistribution of AuNP-liposomes, treatment-related morphological or hemodynamic changes of tumor, or tumor suppression indices (volume and metastasis reduction) of luminescent cancer cell line xenografts. We anticipate future cancer theragnosis, such as a laparoscopic treatment for pre-operation of tumors before their surgical removal, combining the personalized chemotherapy system (in which tunable drug delivery is adjusted by real-time minimally invasive drug-induced biomarker monitoring) to optimize therapeutic efficacy. The fiber-optic cable of laparoscopy for viewing of the affected area from distant can be applied to convey the laser excitation for triggered release of the AuNP-liposome and returning fluorescence signal for assessing drug-induced apoptotic activity.

\section{Conclusion}

We report in vitro and in vivo characterizations of a proposed tunable chemotherapy system with hair-size microcapillaries delivering photo-thermally responsive liposomes directly into tumors and fiber-optic guided laser excitation to trigger the tunable release of these liposomes. The content release profile was quantified by the increase of fluorescence resulting from the dilution of the released self-quenching fluorescein encapsulated in liposomes. In in vitro studies indicated that AuNP-embedded liposomes triggered more efficient triggered release than the traditional temperature-responsive liposome. Using in vivo mouse xenograft studies demonstrated that the encapsulation of fluorescein in liposomes resulted in a more substantial compound retention in the tumor and suggested the tunable release capability of the system by triggering consecutive releases with fiber-optic guided laser excitations.

\section{Acknowledgments}

The authors wish to thank Chief Dr Chun-Ru Dai (R\&D Department, China Medical University, Taichung, Taiwan) for his kindly assistance with in vivo fluorescence imaging acquisition. The research was funded by National Science Council of Taiwan (NSC 97-2218-E-005-096).

\section{Disclosure}

The authors report no conflicts of interest in this work.

\section{References}

1. Qin YY, Li H, Guo XJ, et al. Adjuvant chemotherapy, with or without taxanes, in early or operable breast cancer: a meta-analysis of 19 randomized trials with 30,698 patients. PLoS One. 2011;6(11):e26946.

2. Kim R, Osaki A, Toge T. Current and future roles of neoadjuvant chemotherapy in operable breast cancer. Clin Breast Cancer. 2005;6(3): 223-232.

3. Swain SM. Chemotherapy: updates and new perspectives. Oncologist 2011;16(supp 1):30-39.

4. Dickerson EB, Dreaden EC, Huang X, et al. Gold nanorod assisted near-infrared plasmonic photothermal therapy (PPTT) of squamous cell carcinoma in mice. Cancer Lett. 2008;269(1):57-66. 
5. Hazard HW, Gorla SR, Scholtens D, Kiel K, Gradishar WJ, Khan SA. Surgical resection of the primary tumor, chest wall control, and survival in women with metastatic breast cancer. Cancer. 2008;113(8): 2011-2019.

6. Gnerlich J, Jeffe DB, Deshpande AD, Beers C, Zander C, Margenthaler JA. Surgical removal of the primary tumor increases overall survival in patients with metastatic breast cancer: analysis of the 1988-2003 SEER data. Ann Surg Oncol. 2007;14(8):2187-2194.

7. Rapiti E, Verkooijen HM, Vlastos G, et al. Complete excision of primary breast tumor improves survival of patients with metastatic breast cancer at diagnosis. J Clin Oncol. 2006;24(18):2743-2749.

8. Gurney H. How to calculate the dose of chemotherapy. Br J Cancer. 2002;86(8):1297-1302.

9. Hunter RJ, Navo MA, Thaker PH, Bodurka DC, Wolf JK, Smith JA. Dosing chemotherapy in obese patients: actual versus assigned body surface area (BSA). Cancer Treat Rev. 2009;35(1):69-78.

10. Zhang L, Gu FX, Chan JM, Wang AZ, Langer RS, Farokhzad OC. Nanoparticles in medicine: therapeutic applications and developments. Clin Pharmacol Ther. 2008;83(5):761-769.

11. O'Brien ME, Wigler N, Inbar M, et al. Reduced cardiotoxicity and comparable efficacy in a phase III trial of pegylated liposomal doxorubicin $\mathrm{HCl}$ $\left(\right.$ CAELYX ${ }^{\mathrm{TM}} /$ Doxil $\left.^{\circledR}\right)$ versus conventional doxorubicin for first-line treatment of metastatic breast cancer. Ann Oncol. 2004;15(3):440-449.

12. Gordon AN, Fleagle JT, Guthrie D, Parkin DE, Gore ME, Lacave AJ. Recurrent epithelial ovarian carcinoma: a randomized phase III study of pegylated liposomal doxorubicin versus topotecan. J Clin Oncol. 2001; 19(14):3312-3322.

13. Lyass O, Uziely B, Ben-Yosef R, et al. Correlation of toxicity with pharmacokinetics of pegylated liposomal doxorubicin (Doxil) in metastatic breast carcinoma. Cancer. 2000;89(5):1037-1047.

14. Dos Santos N, Allen C, Doppen AM, et al. Influence of poly(ethylene glycol) grafting density and polymer length on liposomes: relating plasma circulation lifetimes to protein binding. Biochim Biophys Acta. 2007; 1768(6):1367-1377.

15. Gabizon AA. Liposome circulation time and tumor targeting: implications for cancer chemotherapy. Adv Drug Deliv Rev. 1995;16(2-3):285-294.

16. Alaouie AM, Sofou S. Liposomes with triggered content release for cancer therapy. J Biomed Nanotechnol. 2008;4(3):234-244.

17. Kong G, Anyarambhatla G, Petros WP, et al. Efficacy of liposomes and hyperthermia in a human tumor xenograft model: importance of triggered drug release. Cancer Res. 2000;60(24):6950-6957.

18. Huang SK, Stauffer PI, Hong K, et al. Liposomes and hyperthermia in mice: increased tumor uptake and therapeutic efficacy of doxorubicin in sterically stabilized liposomes. Cancer Res. 1994;54(8):2186-2191.

19. Zasadzinski JA, Wong B, Forbes N, Braun G, Wu G. Novel methods of enhanced retention in and rapid, targeted release from liposomes. Curr Opin Colloid Interface Sci. 2011;16(3):203-214.

20. Abraham SA, Waterhouse DN, Mayer LD, Cullis PR, Madden TD, Bally MB. The liposomal formulation of doxorubicin. Methods Enzymol. 2005;391:71-97.

21. Bibi S, Lattmann E, Mohammed AR, Perrie Y. Trigger release liposome systems: local and remote controlled delivery. J Microencapsul. 2012;29(3):262-276.

22. O'Kelly J, Liao KC, Clifton W, Lu D, Koeffler P, Loeb G. Percutaneous fiber-optic sensor for the detection of chemotherapy-induced apoptosis in vivo. Proc SPIE. 2010;7555:75551H.

23. Liao KC, Chiu HS, Fan SY, Zheng YD, Lu PH. Percutaneous fiber-optic biosensor for immediate evaluation of chemotherapy efficacy in vivo (part I): Strategy of assay design for monitoring non-homogeneously distributed biomarkers. Sensor Actuat B-Chemical. 2015.
24. Fan SY, Chiu HS, Lin TY, Liao A, Liao KC. Percutaneous fiberoptic biosensor for immediate evaluation of chemotherapy efficacy in vivo (part II): In vitro and in vivo characterizations. Sensor Actuat B-Chemical. 2015.

25. Derfus AM, von Maltzahn G, Harris TJ, et al. Remotely triggered release from magnetic nanoparticles. Adv Mater. 19(22):3932-3936.

26. Needham D, Dewhirst MW. The development and testing of a new temperature-sensitive drug delivery system for the treatment of solid tumors. Adv Drug Deliv Rev. 2001;53(3):285-305.

27. Needham D, Anyarambhatla G, Kong G, Dewhirst MW. A new temperature-sensitive liposome for use with mild hyperthermia: characterization and testing in a human tumor xenograft model. Cancer Discov. 2000;60(5):1197-1201.

28. Liao KC, Hogen-Esch T, Richmond FJ, Marcu L, Clifton W, Loeb GE. Percutaneous fiber-optic sensor for chronic glucose monitoring in vivo. Biosens Bioelectron. 2008;23(10):1458-1465.

29. Liao KC, Chang SC, Chiu CY, Chou YH. Acute response in vivo of a fiber-optic sensor for continuous glucose monitoring from canine studies on point accuracy. Sensors. 2010;10(8):7789-7802.

30. Agostinis P, Berg K, Cengel KA, et al. Photodynamic therapy of cancer: an update. CA Cancer J Clin. 2011;61(4):250-281.

31. Li JL, Gu M. Gold-nanoparticle-enhanced cancer photothermal therapy. IEEE J Top Quantum Electron. 2010;16(4):989-996.

32. Patra CR, Bhattacharya R, Mukhopadhyay D, Mukherjee P. Fabrication of gold nanoparticles for targeted therapy in pancreatic cancer. $A d v$ Drug Deliv Rev. 2010;62(3):346-361.

33. Chang HI, Yeh MK. Clinical development of liposome-based drugs: formulation, characterization, and therapeutic efficacy. Int J Nanomedicine. 2012;7:49-60.

34. Weinstein JN, Yoshikami S, Henkart P, Blumenthal R, Hagins WA. Liposome-cell interaction: transfer and intracellular release of a trapped fluorescent marker. Science. 1977;195(4277):489-492.

35. Jana NR, Peng X. Single-phase and gram-scale routes toward nearly monodisperse Au and other noble metal nanocrystals. J Am Chem Soc. 2003;125(47):14280-14281.

36. Liu X, Atwater M, Wang J, Huo Q. Extinction coefficient of gold nanoparticles with different sizes and different capping ligands. Colloids Surface B Biointerfaces. 2007;58(1):3-7.

37. Hope MJ, Bally MB, Webb G, Cullis PR. Production of large unilamellar vesicles by a rapid extrusion procedure. Characterization of size, trapped volume and ability to maintain a membrane potential. Biochim Biophys Acta. 1985;812(1):55-65.

38. MacDonald RC, MacDonald RI, Menco BP, Takeshita K, Subbarao NK, Hu LR. Small-volume extrusion apparatus for preparation of large, unilamellar vesicles. Biochim Biophys Acta. 1991;1061(2): 297-303.

39. Paasonen L, Laaksonen T, Johans C, Yliperttula M, Kontturi K, Urtti A. Gold nanoparticles enable selective light-induced contents release from liposomes. J Control Release. 2007;122(1):86-93.

40. Wu G, Mikhailovsky A, Khant HA, Fu C, Chiu W, Zasadzinski JA. Remotely triggered liposome release by near-infrared light absorption via hollow gold nanoshells. J Am Chem Soc. 2008;130(26): 8175-8177.

41. Troutman TS, Leung SJ, Romanowski M. Light-induced content release from plasmon-resonant liposomes. Adv Mater. 2009;21(22): 2334-2338.

42. Leung SJ, Kachur XM, Bobnick MC, Romanowski M. Wavelengthselective light-induced release from plasmon resonant liposomes. $A d v$ Funct Mater. 2011;21(6):1113-1121. 
International Journal of Nanomedicine

Dovepress

\section{Publish your work in this journal}

The International Journal of Nanomedicine is an international, peerreviewed journal focusing on the application of nanotechnology in diagnostics, therapeutics, and drug delivery systems throughout the biomedical field. This journal is indexed on PubMed Central, MedLine, CAS, SciSearch ${ }^{\circledR}$, Current Contents ${ }^{\circledR} /$ Clinical Medicine,
Journal Citation Reports/Science Edition, EMBase, Scopus and the Elsevier Bibliographic databases. The manuscript management system is completely online and includes a very quick and fair peer-review system, which is all easy to use. Visit http://www.dovepress.com/ testimonials.php to read real quotes from published authors.

Submit your manuscript here: http://www.dovepress.com/international-journal-of-nanomedicine-journal 